\title{
Correction
}

\section{Correction: Tanida et al. Comparative Assessment of In-House Real-Time PCRs Targeting Enteric Disease-Associated Microsporidia in Human Stool Samples. Pathogens 2021, 10, 656}

Konstantin Tanida ${ }^{1}$, Andreas Hahn ${ }^{2}$ (D), Kirsten Alexandra Eberhardt ${ }^{3,4} \mathbb{D}^{D}$, Egbert Tannich ${ }^{5,6} \mathbb{D}^{\mathbb{D}}$, Olfert Landt $^{7}$, Simone Kann ${ }^{8}$, Torsten Feldt ${ }^{9}$, Fred Stephen Sarfo ${ }^{10}$, Veronica Di Cristanziano ${ }^{11}$ (D), Hagen Frickmann ${ }^{1,2,+}(\mathbb{D})$ and Ulrike Loderstädt ${ }^{12, *,+}$

Citation: Tanida, K.; Hahn, A.; Eberhardt, K.A.; Tannich, E.; Landt, O.; Kann, S.; Feldt, T.; Sarfo, F.S.; Di Cristanziano, V.; Frickmann, H.; et al. Correction: Tanida et al. Comparative Assessment of In-House Real-Time PCRs Targeting Enteric

Disease-Associated Microsporidia in Human Stool Samples. Pathogens 2021, 10, 656. Pathogens 2022, 11, 256. https://doi.org/10.3390/ pathogens11020256

Received: 24 December 2021 Accepted: 9 February 2022 Published: 17 February 2022

Publisher's Note: MDPI stays neutral with regard to jurisdictional claims in published maps and institutional affiliations.

Copyright: (C) 2022 by the authors. Licensee MDPI, Basel, Switzerland. This article is an open access article distributed under the terms and conditions of the Creative Commons Attribution (CC BY) license (https:// creativecommons.org/licenses/by/ $4.0 /)$.
1 Department of Microbiology and Hospital Hygiene, Bundeswehr Hospital Hamburg, 20359 Hamburg, Germany; konstantintanida@bundeswehr.org (K.T.); frickmann@bnitm.de (H.F.)

2 Department of Medical Microbiology, Virology and Hygiene, University Medicine Rostock, 18057 Rostock, Germany; hahn.andreas@me.com

3 Department of Tropical Medicine, Bernhard Nocht Institute for Tropical Medicine \& I. Department of Medicine, University Medical Center Hamburg-Eppendorf, 20251 Hamburg, Germany; k.eberhardt@bnitm.de

4 Institute for Transfusion Medicine, University Medical Center Hamburg-Eppendorf, 20251 Hamburg, Germany

5 Bernhard Nocht Institute for Tropical Medicine Hamburg, 20359 Hamburg, Germany; tannich@bnitm.de

6 National Reference Centre for Tropical Pathogens, 20359 Hamburg, Germany

7 TIB MolBiol, 12103 Berlin, Germany; oLandt@tib-molbiol.de

8 Medical Mission Institute, 97074 Würzburg, Germany; simone_kann@hotmail.com

9 Department of Gastroenterology, Hepatology and Infectious Diseases, University Medical Center Düsseldorf, 40225 Düsseldorf, Germany; torsten.feldt@med.uni-duesseldorf.de

10 Department of Medicine, Kwame Nkrumah University of Science and Technology, Kumasi, Ghana; stephensarfo78@gmail.com

11 Institute of Virology, Faculty of Medicine and University Hospital of Cologne, University of Cologne, 50935 Cologne, Germany; veronica.di-cristanziano@uk-koeln.de

12 Department of Hospital Hygiene \& Infectious Diseases, University Medicine Göttingen, 37075 Göttingen, Germany

* Correspondence: ulrike.loderstaedt1@med.uni-goettingen.de; Tel.: +49-551-3965709

+ These authors contributed equally to this work.

In the original publication [1], there was a mistake in Table 2 as published. All oligonucleotides of PCR 1 in Table 2 had erroneously been printed in reverse-complement style. The corrected Table 2 appears below. The authors apologize for any inconvenience caused and state that the scientific conclusions are unaffected. The original publication has also been updated. 
Table 2. Details of the real-time PCR assays 1-6, which were included in the test comparison without a reference standard with perfect accuracy for the diagnosis of microsporidia in stool samples. Positive control plasmid inserts are provided in Appendix A.

\begin{tabular}{|c|c|c|c|c|c|c|}
\hline & PCR 1 & PCR 2 & PCR 3 & PCR 4 & PCR 5 & PCR 6 \\
\hline $\begin{array}{l}\text { Target } \\
\text { speci- } \\
\text { ficity }\end{array}$ & $\begin{array}{l}\text { Small subunit } \\
\text { ribosomal RNA } \\
\text { gene of } \\
\text { Enterocytozoon } \\
\text { bieneusi, } \\
\text { Encephalitozoon } \\
\text { cuniculi, } \\
\text { Encephalitozoon } \\
\text { hellem, and } \\
\text { Encephalitozoon } \\
\text { intestinalis }\end{array}$ & $\begin{array}{l}\text { Small subunit } \\
\text { ribosomal RNA } \\
\text { gene of } \\
\text { Enterocytozoon } \\
\text { bieneusi, } \\
\text { Encephalitozoon } \\
\text { cuniculi, } \\
\text { Encephalitozoon } \\
\text { hellem, and } \\
\text { Encephalitozoon } \\
\text { intestinalis }\end{array}$ & $\begin{array}{l}\text { Small subunit } \\
\text { ribosomal RNA } \\
\text { gene of } \\
\text { Enterocytozoon } \\
\text { bieneusi }\end{array}$ & $\begin{array}{c}\text { Internal } \\
\text { transcribed spacer } \\
\text { (ITS) sequence of } \\
\text { Enterocytozoon } \\
\text { bieneusi }\end{array}$ & $\begin{array}{l}\text { Small subunit } \\
\text { ribosomal RNA } \\
\text { gene of } \\
\text { Encephalitozoon } \\
\text { cuniculi, } \\
\text { Encephalitozoon } \\
\text { hellem, and } \\
\text { Encephalitozoon } \\
\text { intestinalis }\end{array}$ & $\begin{array}{c}\text { Internal transcribed } \\
\text { spacer (ITS) } \\
\text { sequence of the } \\
\text { non-target } \\
\text { microorganism } \\
\text { Microsporidium spp. }\end{array}$ \\
\hline $\begin{array}{l}\text { Amplicon } \\
\text { length }\end{array}$ & 394 base pairs & 280 base pairs & 202 base pairs & 105 base pairs & 227 base pairs & 87 base pairs \\
\hline $\begin{array}{l}\text { Cycle } \\
\text { number }\end{array}$ & 50 & 40 & 40 & 50 & 40 & 45 \\
\hline $\begin{array}{l}\text { Forward } \\
\text { primer } 1\end{array}$ & $\begin{array}{c}5^{\prime} \text {-CACCAGG } \\
\text { TTGATTC } \\
\text { TGCCTGA-3' }^{\prime}\end{array}$ & $\begin{array}{l}5^{\prime} \text {-CAGGTT } \\
\text { GATTCTGC } \\
\text { CTGACG-3' }\end{array}$ & $\begin{array}{c}\text { 5'-CCAGGGT }^{\prime} \text { CAAGTCA } \\
\text { TTCGTT- }^{\prime}\end{array}$ & $\begin{array}{c}\text { 5'-TGTGTAG } \\
\text { GCGTGAGA } \\
\text { GTGTATCTG-3' }\end{array}$ & $\begin{array}{c}5^{\prime} \text {-CACCAGG } \\
\text { TTGATTC } \\
\text { TGCCTGAC-3' }\end{array}$ & $\begin{array}{l}5^{\prime} \text {-TCTTGCG } \\
\text { CGTTAAT } \\
\text { GATCCTT-3 }^{\prime}\end{array}$ \\
\hline $\begin{array}{l}\text { Forward } \\
\text { primer } 2\end{array}$ & $\begin{array}{c}\text { 5'-TCCGGAG }^{\prime} \\
\text { AGGGAG } \\
\text { CCTGAG-3' }\end{array}$ & n.a. & n.a. & n.a. & n.a. & n.a. \\
\hline $\begin{array}{l}\text { Reverse } \\
\text { primer } 1\end{array}$ & $\begin{array}{c}5^{\prime}-\mathrm{GCTTGCC} \\
\text { CTCCAAT } \\
\text { TGCTTC-3' }^{\prime}\end{array}$ & $\begin{array}{c}5^{\prime} \text {-CCATCTC } \\
\text { TCAGGCT } \\
\text { CCСTC-3' }\end{array}$ & $\begin{array}{c}5^{\prime} \text {-TATTGTA } \\
\text { TTGCGC } \\
\text { TTGCTGC-3' }^{\prime}\end{array}$ & $\begin{array}{c}\text { 5'-CATCCAA } \\
\text { CCATCACG } \\
\text { TACCAATC-3' }\end{array}$ & $\begin{array}{c}\text { 5'-CTAGTTA } \\
\text { GGCCATTACCC } \\
\text { TAACTACCA-3 }\end{array}$ & $\begin{array}{l}5^{\prime}-\mathrm{AGGTTGC} \\
\text { GGGCGGC-3' }^{\prime}\end{array}$ \\
\hline $\begin{array}{l}\text { Reverse } \\
\text { primer } 2\end{array}$ & $\begin{array}{c}5^{\prime}-\mathrm{GACTTGC} \\
\text { CCTCCAA } \\
\text { TCACATG-3' }^{\prime}\end{array}$ & n.a. & n.a. & n.a. & n.a. & n.a. \\
\hline $\begin{array}{l}\text { Reverse } \\
\text { primer } 3\end{array}$ & $\begin{array}{l}5^{\prime} \text {-CCGACTT } \\
\text { GCCCTCC } \\
\text { AGTAAA-3' }\end{array}$ & n.a. & n.a. & n.a. & n.a. & n.a. \\
\hline $\begin{array}{l}\text { Reverse } \\
\text { primer } 4\end{array}$ & $\begin{array}{c}5^{\prime}-\mathrm{CTTGGCC} \\
\text { TCCAATC } \\
\text { АATCTCG-3' }\end{array}$ & n.a. & n.a. & n.a. & n.a. & n.a. \\
\hline $\begin{array}{l}\text { Hybrid } \\
\text { ization } \\
\text { probe * }\end{array}$ & $\begin{array}{c}\text { 5'-TGGCAGC } \\
\text { AGGCGCG } \\
\text { AAACTTGT-3' }\end{array}$ & n.a. & $\begin{array}{c}5^{\prime}-\text { GATGCCC } \\
\text { TTAGATA } \\
\text { TCCTGG-3' }\end{array}$ & $\begin{array}{l}5^{\prime} \text {-CACTGCA } \\
\text { CCСАCATCC } \\
\text { CTCACССTT-3' }^{\prime}\end{array}$ & $\begin{array}{c}5^{\prime}-\mathrm{CTATCAC} \\
\mathrm{TGAG}+\mathrm{C}+\mathrm{C} \\
\mathrm{GT}+\mathrm{CC}-3^{\prime}\end{array}$ & $\begin{array}{c}\text { 5'-ACGGAAGA } \\
\text { GCTTCGG } \\
\text { GGGCCA-3' }\end{array}$ \\
\hline
\end{tabular}

n.a. $=$ not applicable. ${ }^{*}$ Bases with a plus (+) in front of them are locked nucleic acid (LNA) bases.

\section{Reference}

1. Tanida, K.; Hahn, A.; Eberhardt, K.A.; Tannich, E.; Landt, O.; Kann, S.; Feldt, T.; Sarfo, F.S.; Di Cristanziano, V.; Frickmann, H.; et al. Comparative Assessment of In-House Real-Time PCRs Targeting Enteric Disease-Associated Microsporidia in Human Stool Samples. Pathogens 2021, 10, 656. [CrossRef] [PubMed] 